\section{Autoavaliação do estado de saúde e a associação com fatores sociodemográficos, hábitos de vida e morbidade na população: um inquérito nacional}

\author{
Self-rated health and the association with social \\ and demographic factors, health behavior, and \\ morbidity: a national health survey
}

Autoevaluación del estado de salud y la asociación con factores sociodemográficos, hábitos de vida y morbilidad en la población: una encuesta nacional

\footnotetext{
${ }^{1}$ Instituto de Medicina Social, Universidade do Estado do Rio de Janeiro, Rio de Janeiro, Brasil. 2 Escola Nacional de Saúde Pública Sergio Arouca, Fundação Oswaldo Cruz, Rio de Janeiro, Brasil.

Correspondência A. L. B. Pavão Instituto de Medicina Social, Universidade do Estado do Rio de Janeiro.

Rua São Francisco Xavier 524, 7 o andar, Rio de Janeiro, $R J$ 20559-900, Brasil. analuizabp@gmail.com
}

\begin{abstract}
Self-rated health is an important indicator used to measure health perception. This study aimed to investigate the association between self-rated health and social and demographic factors, health behavior, and morbidity. This was a crosssectional study based on data from a national health survey. The sample consisted of 12,324 individuals from Brazil's five major geographic regions. Regression analyses were conducted to verify the association between the outcome and the following independent variables: gender, age, income, education, race, marital status, smoking, alcohol consumption, physical activity, chronic illnesses, and body mass index. Increasing age, low education and income, smoking, sedentary habits, chronic illness, and obesity were the factors most strongly associated with worse self-rated health. The identification of population attributes associated with worse self-rated health can help trace a profile of individuals more prone to seeking health services.
\end{abstract}

Diagnostic Self Evaluation; Morbidity; Epidemiologic Factors; Health Surveys
Ana Luiza Braz Pavão 1 Guilherme Loureiro Werneck 1 Mônica Rodrigues Campos 2

\section{Resumo}

A autoavaliação do estado de saúde é um importante indicador da percepção de saúde. Este estudo visa investigar a associação entre autoavaliação de saúde e fatores sociodemográficos, hábitos de vida e morbidade na população brasileira. Trata-se de estudo seccional, baseado num inquérito nacional, que avaliou 12.324 indivíduos, nas cinco regiões geográficas do país. Foram aplicados modelos de regressão para avaliar a associação entre o desfecho, avaliado de forma dicotômica, e variáveis de exposição: sexo, idade, renda, escolaridade, raça, situação conjugal, tabagismo, atividade física, consumo de álcool, presença de morbidade crônica e índice de massa corporal. O aumento da idade, possuir baixos níveis de escolaridade e renda, tabagismo, sedentarismo, presença de morbidade crônica e obesidade foram os principais fatores associados à autoavaliação ruim. A identificação das características da população que estão associadas a um pior estado de saúde percebido pode contribuir para traçar um perfil dos indivíduos mais propensos a procurar os serviços de saúde.

Auto-avaliação Diagnóstica; Morbidade; Fatores Epidemiológicos; Inquéritos Epidemiológicos 


\section{Introdução}

O conceito de saúde apresenta múltiplas dimensões. Pode ser entendido, por exemplo, como "o estado do indivíduo cujas funções orgânicas, físicas e mentais se acham em situação normal; o estado do que é sadio ou são" 1 (p. 1556). Uma revisão sobre os principais modelos para a conceituação de saúde revelou a complexidade desse construto e identificou, dentre outras, duas principais definições: a primeira, definindo saúde como a ausência de doença ou incapacidade; a segunda, considerando saúde o estado de completo bem-estar físico, mental e social, e não apenas a ausência de doença 2 . Em termos operacionais, tal construto pode ser medido por meio de informações médicas diretas, que são baseadas em sinais e sintomas patológicos e em exames diagnósticos; ou indiretas, por meio dos registros médicos. Pode ainda ser mensurado por avaliações feitas pelos próprios indivíduos, ou morbidade referida, utilizando-se de diferentes indicadores, como a autoavaliação do estado de saúde, o número de sintomas ou de doenças crônicas relatadas, ou o número de dias em que o paciente apresentou restrição de suas atividades habituais por conta de doença 3 .

A autoavaliação do estado de saúde é um importante indicador do construto multidimensional da saúde e consiste na percepção que os indivíduos possuem de sua própria saúde. É, portanto, um indicador subjetivo e engloba tanto componentes físicos quanto emocionais dos indivíduos, além de aspectos do bem-estar e da satisfação com a própria vida. A percepção do paciente de se sentir doente não advém apenas das sensações físicas de dor e desconforto, mas sobretudo, das consequências sociais e psicológicas da presença da enfermidade ${ }^{4}$. Este indicador vem sendo amplamente utilizado em estudos epidemiológicos, tanto pela sua relativa facilidade de captação em inquéritos populacionais, como também pela sua validade, sendo importante preditor de morbidade e de mortalidade e apresentando associação com determinadas condições clínicas 5,6. Também foi verificada boa concordância desse indicador com a avaliação médica do estado de saúde ${ }^{7}$. Além disso, alguns estudos mostram que pode ser ainda um importante fator na predição de problemas de saúde que só seriam detectados mais tardiamente 8,9. Dessa forma, vem sendo aplicado com a finalidade de estabelecer diferenças na morbidade entre subgrupos populacionais e comparar a necessidade por serviços de saúde e por recursos entre diferentes áreas geográficas.

Entre os determinantes da autoavaliação do estado de saúde, os achados de inquéritos realiza- dos no Brasil sugerem que a idade, o sexo, o bemestar material e o grau de instrução são dimensões de relevância ${ }^{4}$. Além disso, a situação de trabalho, as características do estilo de vida, como a prática de exercícios físicos, o tabagismo e o consumo de álcool, bem como os fatores psicossociais também parecem estar envolvidos e influenciam a percepção que os indivíduos têm de sua saúde, segundo estudos conduzidos em diferentes países 10,11,12,13,14,15,16,17. Apesar dessas evidências, poucos estudos foram conduzidos no Brasil com a finalidade de analisar os fatores envolvidos na autoavaliação do estado de saúde dos indivíduos. Foram encontrados apenas três estudos, sendo um deles baseado em dados da Pesquisa Nacional por Amostra de Domicílios (PNAD 2003), outro em dados da Pesquisa Mundial de Saúde (2003) e um terceiro utilizando dados de um inquérito por telefone $4,18,19$. Os dois primeiros avaliaram apenas a associação com fatores sociodemográficos. O terceiro, apesar de ter investigado também hábitos de vida e morbidade, avaliou apenas os indivíduos residentes em moradias com linha de telefonia fixa. Sendo assim, o objetivo do presente artigo é, inicialmente, estimar a prevalência de autoavaliação do estado de saúde ruim na população brasileira e, em seguida, investigar sua associação com fatores sociodemográficos, hábitos de vida e morbidade, na população que participou de um inquérito domiciliar de abrangência nacional.

\section{Métodos}

\section{Delineamento do estudo e amostragem}

O estudo possui um delineamento seccional, com base em dados de um inquérito domiciliar de abrangência nacional, realizado em 2008, nas cinco regiões geográficas brasileiras, denominado Pesquisa Dimensão Social das Desigualdades (PDSD). Esse estudo teve como objetivo principal propor um sistema de indicadores sociológicos para o acompanhamento da dinâmica da desigualdade e da mobilidade social no Brasil.

Estudou-se uma amostra de 8.048 domicílios, com representatividade nacional, obtidos pela amostragem estratificada por conglomerados, em três estágios (microrregião, setor censitário e domicílio). A unidade de análise foi o domicílio. A população foi subdividida em seis domínios, definidos de acordo com a região geográfica (Norte, Nordeste, Centro-oeste, Sul e Sudeste) e situação (urbano e rural). Foi criado estrato amostral com os $10 \%$ mais ricos dos setores censitários para a melhor precisão dos indicadores de desigualdade. Um total de 12.324 chefes de família e cônjuges, com idade maior ou igual a 20 anos, 
foram entrevistados por meio de questionários e avaliação de medidas antropométricas. A coleta de dados durou seis meses e foi realizada por uma equipe do Instituto Brasileiro de Opinião Pública e Estatística (IBOPE), constituída por entrevistadores, críticos, revisores e supervisores. Houve muitos casos de descartes de domicílios da amostra por não ser possível realizar a segunda entrevista (chefe ou cônjuge), especialmente pela recusa de um deles. Um dos motivos das recusas era o tamanho do questionário/duração da entrevista. Quando não era possível a entrevista com o chefe e o cônjuge, o domicílio era desconsiderado para a amostra. Duas estratégias foram adotadas pela equipe de trabalho de campo para melhorar a produtividade: o envio de uma dupla de entrevistadores e a realização das entrevistas em cômodos diferentes do domicílio, o que reduziu o tempo total gasto pelas entrevistas e contribuiu para a diminuição das recusas. Houve 571 domicílios considerados inelegíveis e cerca de $20 \%$ foram substituídos por outros (domicílios mais próximos), a fim de que todos os domicílios previstos para o estudo fossem entrevistados.

\section{Instrumento de avaliação}

O questionário apresentava as seguintes dimensões: características do domicílio (estrutura); características gerais do chefe e cônjuge; características dos pais, irmãos e amigos; atividade econômica; procura de trabalho; saúde; antropometria; fecundidade; avaliação das condições de vida; percepções de justiça; experiência de discriminação. Os módulos do questionário cujas informações serão utilizadas na análise do presente estudo são: "características gerais do chefe e cônjuge" e "características do domicílio (estrutura)", para a obtenção dos dados sociodemográficos, o módulo "saúde", para a obtenção de informações sobre o desfecho (autoavaliação do estado de saúde) e sobre as variáveis relativas a hábitos de vida e comportamentos relacionados à saúde, e, finalmente, o módulo de "antropometria", para o cálculo do índice de massa corporal (IMC). Como o inquérito PDSD é um estudo que busca avaliar as características da mobilidade social no Brasil, alguns módulos específicos do questionário (incluindo o módulo "saúde") foram respondidos apenas pelo chefe de família, pois constituem a força produtiva de uma sociedade e, em alguns casos, também foram respondidos pelos cônjuges, para avaliar características do casal. No presente estudo, optou-se por analisar as informações relativas aos chefes e cônjuges, pois o objetivo era avaliar a situação de saúde de todos os indivíduos que responderam a esse módulo do questionário.

\section{Variáveis de exposição e desfecho}

O desfecho avaliado foi a autoavaliação do estado de saúde, obtido pela seguinte pergunta, localizada no início do módulo "saúde" do questionário, baseada em questão contida no instrumento de qualidade de vida Short-Form-36 (SF-36), adaptado para o português: "Em geral, você diria que sua saúde é:", com as seguintes opções de resposta: "excelente", "muito boa", "boa", "razoável" e "ruim". As cinco categorias iniciais de resposta, para o desfecho da autoavaliação do estado de saúde, foram dicotomizadas em: boa (categorias "excelente", "muito boa" e "boa") e ruim (categorias "razoável" e "ruim").

Foram investigadas as seguintes variáveis como potenciais determinantes da autoavaliação do estado de saúde: sociodemográficas (idade, sexo, renda domiciliar, escolaridade, situação conjugal e raça); as relacionadas aos hábitos de vida e comportamentos relacionados à saúde (tabagismo, prática de atividades físicas e consumo de álcool) e características relativas à morbidade (avaliada pela presença de doenças crônicas relatadas pelo indivíduo e pelo IMC).

A idade, em anos, foi categorizada em três faixas etárias, a saber: até 39 anos, de 40 a 64 anos e $\geq 65$ anos. A renda utilizada foi a per capita domiciliar, categorizada de acordo com o número de salários mínimos (SM), da seguinte maneira: $\leq$ 0,5SM, 0,5-1SM, 1-1,5SM, 1,5-2SM e > 2SM. A variável escolaridade foi analisada de acordo com as seguintes categorias: analfabeto, 1 a 4 anos de estudo, 5 a 8 anos de estudo, segundo grau e nível superior. A informação sobre raça foi obtida mediante pergunta ao entrevistado sobre como ele classificaria a sua cor de pele. As opções de resposta no questionário eram: branca, preta, parda, amarela e indígena. Optou-se por avaliar apenas as categorias de brancos, pretos e pardos por constituírem cerca de $97 \%$ dos indivíduos da amostra. Em relação aos hábitos de vida, o tabagismo foi classificado em três categorias: nunca fumou, já fumou mas não fuma mais e fuma atualmente. Atividade física foi avaliada nas seguintes categorias: sentado a maior parte do dia, não anda muito durante o dia, caminha ou fica de pé bastante durante o dia, mas não carrega ou levanta coisas com frequência, carrega pesos leves ou sobe escadas com frequência ou faz exercícios regulares, faz trabalho pesado ou exercícios pesados regularmente. Consumo de álcool foi medido pelos seguintes estratos: não consumiu bebida alcoólica no último ano, consumiu no máximo 1 vez/mês, entre 1 a 4 vezes/mês, mais de 1 vez até 3 vezes/semana, de 4 vezes/semana até diariamente. A presença de doenças crônicas baseouse em uma lista de doenças crônicas presente no 
questionário, em que o entrevistador perguntava se algum médico ou profissional de saúde alguma vez dera o diagnóstico. A lista era composta por 16 doenças, a saber: artrite, câncer, doença cardíaca, diabetes, hipertensão, tuberculose, doenças da coluna, cirrose, gota, tendinite, lesão por esforço repetitivo, bronquite crônica, depressão, doença renal crônica, asma e colesterol alto. Foram criadas quatro categorias para a variável morbidade, de acordo com a presença (ou não) dessas doenças, da seguinte forma: saudável, 1 morbidade relatada, 2 morbidades relatadas, 3 ou mais morbidades relatadas. O IMC, medido $\mathrm{em} \mathrm{kg} / \mathrm{m}^{2}$, foi avaliado nas seguintes categorias: baixo peso $\left(<18,5 \mathrm{~kg} / \mathrm{m}^{2}\right)$, peso adequado (entre $18,5 \mathrm{e}<25 \mathrm{~kg} / \mathrm{m}^{2}$ ), sobrepeso (entre $25 \mathrm{e}<30 \mathrm{~kg}$ / $\left.\mathrm{m}^{2}\right)$ e obeso $\left(\geq 30 \mathrm{~kg} / \mathrm{m}^{2}\right)$.

Algumas categorias de variáveis foram agrupadas por se tratarem de grupos populacionais com hábitos comportamentais semelhantes. No caso da variável "consumo de álcool", as categorias "entre 1 a 4 vezes/mês" e "mais de 1 vez até 3 vezes/semana" foram agrupadas em "de 1 vez/ mês a 3 vezes/semana". E, no caso da variável relativa à atividade física, as categorias "sentado a maior parte do dia" e "não anda muito durante o dia" foram agrupadas na categoria "sedentário" e as categorias "carrega pesos leves ou sobe escadas com frequência ou faz exercícios regulares" e "faz trabalho pesado ou exercícios pesados regularmente" numa única categoria, a saber: "atividade física regular". A categoria "caminha ou fica de pé bastante durante o dia, mas não carrega ou levanta coisas com frequência" foi substituída por "atividade física irregular". A classificação da variável atividade física em sedentário, regular e irregular teve como objetivo simplificar a sua interpretação e facilitar o seu manuseio na análise, agrupando categorias semelhantes.

\section{Análises}

Foram calculadas prevalências de autoavaliação do estado de saúde ruim para todas as categorias das variáveis de exposição. Modelos de regressão logística uni e multivariados foram utilizados, para identificar a associação entre as variáveis de exposição e a autoavaliação do estado de saúde. As variáveis renda e presença de doenças crônicas (morbidade) entraram nos modelos na forma ordinal. As demais variáveis foram avaliadas como categóricas. As associações foram expressas na forma de odds ratio (OR) e os resultados das prevalências apresentados em valores percentuais. Para ambos, foram estimados intervalos de 95\% de confiança (IC95\%). Uma vez que a amostra foi selecionada em múltiplos estágios, a correção para o efeito do delineamento foi realizada por meio do comando svy para análise de dados provenientes de amostras complexas 20 . O programa estatístico utilizado na análise dos dados foi o Stata/IC versão 11 (Stata Corp., College Station, Estados Unidos).

\section{Resultados}

\section{Características gerais da amostra}

A população do estudo era composta, em sua maioria, por mulheres $(57,7 \%)$, com idade entre 40 e 64 anos $(49,4 \%)$ e residentes na Região Sudeste do país $(47,6 \%)$. A maioria possuía de 1 a 4 anos de estudo $(31,1 \%)$ e encontrava-se na categoria de renda igual ou inferior a $0,5 \mathrm{SM}(40 \%)$. Em relação à raça, $48,7 \%$ se autoclassificaram como brancos, $39,8 \%$ como pardos e $11,5 \%$ se consideraram pretos. A prevalência de autoavaliação do estado de saúde ruim na amostra total era de, aproximadamente, 40\% (IC95\%: 38,9-41,0).

As mulheres, os indivíduos com maior idade, pertencentes aos menores níveis de renda e escolaridade, residentes na Região Nordeste do país e viúvos apresentaram maior prevalência de autoavaliação de saúde ruim (Tabela 1).

Os indivíduos que já fumaram ou fumam atualmente tiveram as maiores prevalências de autoavaliação de saúde ruim. Em relação ao consumo de álcool, os extremos para essa categoria apresentaram os maiores valores: a categoria que relatou não consumir bebida alcoólica e aquela que consome de 4 vezes por semana a diariamente. Quanto mais intensa e frequente foi a prática de atividade física, menor a prevalência de autoavaliação de saúde ruim. Em relação à morbidade, quanto maior o número de doenças crônicas, maior a prevalência de autoavaliação de saúde ruim, que também foi verificada mais frequentemente na categoria de obesos e de indivíduos em baixo peso (Tabela 2).

As variáveis sociodemográficas, de hábitos de vida e de morbidade estiveram significativamente associadas com o desfecho, na análise univariada. Em geral, houve uma redução da magnitude dessas associações após ajuste mútuo na análise multivariada. Em relação ao sexo, os resultados indicaram que as mulheres apresentaram $10 \%$ mais chance de ter uma autoavaliação de saúde ruim, quando comparadas aos homens; no entanto, tal efeito foi marginalmente significativo. Também, para raça, o efeito foi marginalmente significativo. Não foi encontrada significância estatística para a associação com situação conjugal (Tabela 3).

Indivíduos pertencentes à maior faixa etária (65 anos ou mais) tiveram chance cerca de 
Tabela 1

Prevalência de autoavaliação do estado de saúde ruim, segundo sexo, idade, escolaridade, renda, situação conjugal, região e raça, na população de 12.324 indivíduos. Pesquisa Dimensão Social das Desigualdades (PDSD), Brasil, 2008.

\begin{tabular}{|c|c|c|c|}
\hline \multirow[t]{2}{*}{ Variáveis } & \multirow[t]{2}{*}{$\mathrm{n}$} & \multicolumn{2}{|c|}{$\begin{array}{l}\text { Prevalênciada de autoavaliação do } \\
\text { estado de saúde ruim }\end{array}$} \\
\hline & & $\%$ & IC95\% \\
\hline \multicolumn{4}{|l|}{ Sexo } \\
\hline Feminino & 7.168 & 44,4 & $43,0-45,9$ \\
\hline Masculino & 5.255 & 33,8 & $32,3-35,4$ \\
\hline \multicolumn{4}{|l|}{ Idade (anos) } \\
\hline Até 39 & 3.973 & 20,3 & $18,8-21,8$ \\
\hline $40-64$ & 6.132 & 44,3 & $42,8-45,8$ \\
\hline 65 ou mais & 2.318 & 62,2 & $59,7-64,5$ \\
\hline \multicolumn{4}{|l|}{ Região } \\
\hline Norte & 595 & 35,9 & $32,1-39,8$ \\
\hline Nordeste & 3.315 & 47,6 & $15,7-49,5$ \\
\hline Cento-oeste & 611 & 35,4 & $31,5-39,5$ \\
\hline Sudeste & 5.912 & 35,3 & $33,7-37,0$ \\
\hline Sul & 1.990 & 43,6 & $41,3-45,8$ \\
\hline \multicolumn{4}{|l|}{ Situação conjugal } \\
\hline Solteiro & 1.171 & 29,7 & $26,6-32,9$ \\
\hline Casado ou morando com companheiro & 8.823 & 37,5 & $36,3-38,8$ \\
\hline Separado & 1.099 & 47,8 & $44,2-51,4$ \\
\hline Viúvo & 1.330 & 58,6 & $55,3-61,8$ \\
\hline \multicolumn{4}{|l|}{ Raça } \\
\hline Branco & 5.868 & 37,3 & $35,8-38,8$ \\
\hline Pardo & 4.801 & 42,3 & $40,6-44,0$ \\
\hline Preto & 1.389 & 43,2 & $40,1-46,5$ \\
\hline \multicolumn{4}{|l|}{ Escolaridade } \\
\hline Analfabeto & 1.904 & 61,7 & $59,0-64,3$ \\
\hline 1-4 anos & 3.592 & 51,5 & $49,5-53,5$ \\
\hline $5-8$ anos & 2.529 & 36,6 & $34,3-38,9$ \\
\hline 2o grau & 2.408 & 23,0 & $20,9-25,1$ \\
\hline Nível superior & 1.109 & 15,6 & $13,1-18,5$ \\
\hline \multicolumn{4}{|l|}{ Renda (salários mínimos) } \\
\hline$\leq 0,5$ & 4.453 & 46,5 & $44,8-48,3$ \\
\hline$>0,5$ e $\leq 1,0$ & 3.619 & 43,6 & $41,6-45,7$ \\
\hline$>1,0$ e $\leq 1,5$ & 1.107 & 33,3 & $29,9-36,9$ \\
\hline$>1,5$ e $\leq 2,0$ & 788 & 25,8 & $22,2-29,7$ \\
\hline$>2,0$ & 1.169 & 22.8 & $20,0-25,8$ \\
\hline
\end{tabular}

IC95\%: intervalo de 95\% de confiança.

$100 \%$ maior de ter autoavaliação de saúde ruim, quando comparados aos indivíduos pertencentes à menor faixa etária (até 39 anos) (IC95\%: $1,67-2,58)$.

Em relação à região de moradia, verificou-se que os indivíduos residentes nas regiões Sudeste e Sul apresentaram significativamente menores chances de ter autoavaliação de saúde ruim, quando comparados aos indivíduos residentes na Região Nordeste.
Em relação à escolaridade, os indivíduos analfabetos tiveram chance aproximadamente $340 \%$ maior de se autoavaliarem com saúde ruim do que os indivíduos que possuíam nível superior (IC95\%: 3,13-6,09). Para cada incremento na faixa de renda, observou-se uma redução de $21 \%$ na chance de relatar autoavaliação de saúde ruim (IC95\%: 0,75-0,84).

Indivíduos que já fumaram e não fumam mais e os que fumam atualmente tiveram sig- 
nificativamente maiores chances de relatar autoavaliação de saúde ruim do que os que nunca fumaram. Em relação ao consumo de álcool, apenas a categoria dos indivíduos que relataram não consumir bebida alcoólica apresentou significativamente mais chance de ter autoavaliação de saúde ruim, quando comparados com consumidores moderados.

Sobre atividade física, indivíduos sedentários e os indivíduos que fazem atividade física irregular tiveram chances de relatar autoavaliação de saúde ruim aproximadamente $80 \%$ e $40 \%$ maiores, respectivamente, do que os indivíduos que exercem atividade física regularmente (IC95\%: 1,50-2,15, para os indivíduos sedentários, e IC95\%: 1,17-1,61, para os indivíduos com atividade física irregular).
Sobre os relatos de morbidade, quanto maior o número de doenças crônicas relatadas, a chance de ter uma autoavaliação de saúde ruim foi progressivamente maior, aumentando em cerca de $140 \%$ de uma categoria para outra (IC95\%: 2,28-2,57).

Em relação ao IMC, indivíduos em baixo peso e em sobrepeso não tiveram resultados estatisticamente significativos após o ajuste. Apenas os indivíduos obesos apresentaram maior chance de ter autoavaliação do estado de saúde ruim, comparados aos indivíduos eutróficos (IC95\%: 1,07-1,49) (Tabela 3).

Tabela 2

Prevalência de autoavaliação do estado de saúde ruim, segundo tabagismo, consumo de álcool, atividade física, presença de morbidade crônica e índice de massa corporal (IMC), na população de 12.324 indivíduos. Pesquisa Dimensão Social das Desigualdades (PDSD), Brasil, 2008.

\begin{tabular}{|c|c|c|c|}
\hline \multirow[t]{2}{*}{ Variáveis } & \multirow[t]{2}{*}{$\mathbf{n}$} & \multicolumn{2}{|c|}{$\begin{array}{c}\text { Prevalênciada de autoavaliação } \\
\text { do estado de saúde ruim }\end{array}$} \\
\hline & & $\%$ & IC95\% \\
\hline \multicolumn{4}{|l|}{ Tabagismo } \\
\hline Nunca fumou & 6.925 & 35,4 & $34,0-36,8$ \\
\hline Já fumou & 3.087 & 48,1 & $45,9-50,2$ \\
\hline Fuma atualmente & 2.411 & 42,7 & $40,3-45,1$ \\
\hline \multicolumn{4}{|l|}{ Consumo de álcool } \\
\hline Não consumiu & 7.576 & 46,4 & $45,0-47,8$ \\
\hline 1 vez por mês & 2.062 & 31,7 & $29,3-34,1$ \\
\hline $1-4$ vezes por semana & 1.607 & 26,2 & $23,6-28,9$ \\
\hline Até 3 vezes por semana & 606 & 27,5 & $23,1-32,3$ \\
\hline De 4 vezes por semana a diariamente & 573 & 36,0 & $31,0-41,3$ \\
\hline \multicolumn{4}{|l|}{ Atividade física } \\
\hline Sentado na maior parte do dia & 2.196 & 49,3 & $46,7-51,9$ \\
\hline Não anda muito durante o dia & 1.469 & 48,7 & $45,5-51,8$ \\
\hline Caminha mas não carrega peso & 6.011 & 39,8 & $38,4-41,4$ \\
\hline Carrega pesos leves ou faz exercícios regulares & 1.786 & 28,0 & $25,5-30,6$ \\
\hline Faz trabalho pesado ou exercícios pesados regularmente & 844 & 28,4 & $24,9-32,2$ \\
\hline \multicolumn{4}{|l|}{ Morbidade } \\
\hline Saudável & 4.554 & 14,2 & $13,1-15,5$ \\
\hline 1 & 3.035 & 37,4 & $35,3-39,5$ \\
\hline 2 & 1.996 & 54,7 & $52,0-57,4$ \\
\hline 3 ou mais & 2.837 & 73,6 & $71,5-75,6$ \\
\hline \multicolumn{4}{|l|}{ IMC } \\
\hline Baixo peso & 417 & 43,6 & $38,0-49,4$ \\
\hline Eutrófico & 5.093 & 34,4 & $32,8-36,0$ \\
\hline Sobrepeso & 4.268 & 40,6 & $38,8-42,4$ \\
\hline Obeso & 2.396 & 48,9 & $46,5-51,4$ \\
\hline
\end{tabular}

IC95\%: intervalo de 95\% de confiança. 
Tabela 3

Odds ratio (OR) bruta e ajustada de autoavaliação do estado de saúde ruim, segundo variáveis sociodemográficas, de hábitos de vida ou comportamentais de saúde e morbidade, na população de 12.324 indivíduos. Pesquisa Dimensão Social das Desigualdades (PDSD), Brasil, 2008.

\begin{tabular}{|c|c|c|c|c|}
\hline Variáveis & OR bruta & IC95\% & OR ajustada * & IC95\% \\
\hline \multicolumn{5}{|l|}{ Sexo } \\
\hline Feminino & 1,00 & - & 1,00 & - \\
\hline Masculino & 1,57 & $1,43-1,72$ & 1,10 & $0,96-1,27$ \\
\hline \multicolumn{5}{|l|}{ Idade (anos) } \\
\hline Até 39 & 1,00 & - & 1,00 & - \\
\hline $40-64$ & 3,13 & $2,80-3,51$ & 1,73 & $1,48-2,02$ \\
\hline 65 ou mais & 6,47 & $5,63-7,43$ & 2,07 & $1,67-2,58$ \\
\hline \multicolumn{5}{|l|}{ Região } \\
\hline Norte & 0,62 & $0,51-0,74$ & 0,81 & $0,63-1,04$ \\
\hline Nordeste & 1,00 & - & 1,00 & - \\
\hline Cento-oeste & 0,60 & $0,50-0,73$ & 0,79 & $0,61-1,04$ \\
\hline Sudeste & 0,60 & $0,54-0,67$ & 0,54 & $0,46-0,63$ \\
\hline Sul & 0,85 & $0,75-0,96$ & 0,63 & $0,52-0,76$ \\
\hline \multicolumn{5}{|l|}{ Situação conjugal } \\
\hline Solteiro & 1,42 & $1,21-1,67$ & 1,02 & $0,81-1,27$ \\
\hline Casado ou morando com companheiro & 1,00 & - & 1,00 & - \\
\hline Separado & 2,17 & $1,76-2,68$ & 1,12 & $0,84-1,49$ \\
\hline Viúvo & 3,35 & $2,74-4,09$ & 0,96 & $0,72-1,28$ \\
\hline \multicolumn{5}{|l|}{ Raça } \\
\hline Branco & 1,00 & - & 1,00 & - \\
\hline Pardo & 1,23 & $1,12-1,36$ & 1,11 & $0,96-1,28$ \\
\hline Preto & 1,28 & $1,11-1,48$ & 1,19 & $0,98-1,46$ \\
\hline \multicolumn{5}{|l|}{ Escolaridade } \\
\hline Analfabeto & 8,70 & $6,91-10,96$ & 4,37 & $3,13-6,09$ \\
\hline $1-4$ anos & 5,74 & $4,62-7,14$ & 4,22 & $3,11-5,73$ \\
\hline $5-8$ anos & 3,12 & $2,49-3,91$ & 2,94 & $2,16-4,00$ \\
\hline 2o Grau & 1,61 & $1,28-2,03$ & 20,1 & $1,48-2,72$ \\
\hline Nível Superior & 1,00 & - & 1,00 & - \\
\hline Renda & 0,76 & $0,73-0,78$ & 0,79 & $0,75-0,84$ \\
\hline \multicolumn{5}{|l|}{ Tabagismo } \\
\hline Nunca fumou & 1,00 & - & 1,00 & - \\
\hline Já fumou & 1,69 & $1,52-1,88$ & 1,25 & $1,07-1,45$ \\
\hline Fuma atualmente & 1,36 & $1,21-1,53$ & 1,36 & $1,16-1,60$ \\
\hline \multicolumn{5}{|l|}{ Consumo de álcool } \\
\hline Não consumiu & 2,47 & $2,11-2,89$ & 1,50 & $1,24-1,80$ \\
\hline 1 vez por mês & 1,28 & $1,06-1,56$ & 1,10 & $0,89-1,36$ \\
\hline De 1 vez por mês até 3 vezes por semana & 1,00 & - & 1,00 & - \\
\hline De 4 vezes por semana até diariamente & 1,52 & $1,14-2,03$ & 1,21 & $0,86-1,69$ \\
\hline \multicolumn{5}{|l|}{ Atividade física } \\
\hline Sedentário & 2,46 & $2,16-2,80$ & 1,80 & $1,50-2,15$ \\
\hline Atividade física irregular & 1,69 & $1,50-1,91$ & 1,37 & $1,17-1,61$ \\
\hline Atividade física regular & 1,00 & - & 1,00 & - \\
\hline Morbidade & 2,50 & $2,39-2,61$ & 2,42 & $2,28-2,57$ \\
\hline \multicolumn{5}{|l|}{ IMC } \\
\hline Baixo peso & 1,47 & $1,15-1,88$ & 1,02 & $0,71-1,47$ \\
\hline Eutrófico & 1,00 & - & 1,00 & - \\
\hline Sobrepeso & 1,30 & $1,17-1,44$ & 1,06 & $0,93-1,23$ \\
\hline Obeso & 1,83 & $1,62-2,06$ & 1,26 & $1,07-1,49$ \\
\hline
\end{tabular}

IC95\%: intervalo de 95\% de confiança; IMC: índice de massa corporal.

* As variáveis foram ajustadas umas pelas outras. 


\section{Discussão}

No presente estudo, o aumento da idade, possuir baixos níveis de escolaridade e renda, tabagismo, sedentarismo, não consumir bebida alcoólica, presença de morbidade crônica e obesidade foram os principais fatores associados à autoavaliação do estado de saúde ruim na população brasileira.

É bem estabelecido que, quanto mais se avança para faixas etárias maiores, mais o estado geral de saúde diminui e, consequentemente, a autoavaliação de saúde decai 21. Como a maioria das doenças crônicas é mais prevalente em faixas etárias mais avançadas, o indicador da autoavaliação de saúde tende a piorar com o aumento da idade, em decorrência do pior estado geral de saúde 6 . Um estudo no Canadá observou que indivíduos com 55 anos ou mais apresentaram $150 \%$ mais chance de relatarem autoavaliação do estado de saúde ruim do que indivíduos com menos de 55 anos 17. No Brasil, dados nacionais da Pesquisa Mundial de Saúde mostraram que o aumento da idade diminuiu a chance de autoavaliação de saúde boa ou muito boa em homens e mulheres, mesmo após o ajuste por variáveis socioeconômicas 4 , o que está de acordo com os resultados do presente estudo. Contudo, existem evidências também de que, entre os indivíduos em idade avançada, quanto maior a idade, melhor a percepção sobre a sua saúde. Esses achados são explicados, segundo os autores, por uma redução nas expectativas com relação à saúde de indivíduos nas últimas fases da vida, pois o fato de terem sobrevivido até aquele estágio já representa um estado de saúde positivo. Também é possível que este resultado tenha sofrido o efeito do viés de sobrevivência seletiva, que decorre de apenas os indivíduos mais sadios terem sobrevivido até as faixas etárias mais avançadas e, por isso, possuírem melhores percepções sobre sua saúde 22. De toda a forma, os achados evidenciam a necessidade de uma maior atenção à saúde da população idosa, o que se torna ainda mais importante pelo evidente processo de envelhecimento populacional no Brasil.

As mulheres tiveram pior avaliação de seu estado de saúde do que os homens, todavia, após o ajuste para as demais variáveis, a associação diminuiu muito e se tornou não estatisticamente significante neste estudo. Segundo dados da literatura, os resultados da influência do sexo na autoavaliação do estado de saúde mostram-se conflitantes 23 . No Canadá, as mulheres apresentaram menor chance de relatar autoavaliação do estado de saúde ruim, quando comparadas aos homens $(\mathrm{OR}=0,63)$ 17. No Brasil, estudo baseado em dados da PNAD encontrou razão de chan- ces $15 \%$ maior para as mulheres, em relação aos homens, de terem autoavaliação de saúde ruim $(\mathrm{p}<0,001)$ 18. Já um estudo realizado na Argentina, mostrou pior autoavaliação de saúde entre as mulheres, porém a diferença não foi estatisticamente significativa 21. Em geral, as mulheres tendem a perceber o seu estado de saúde mais negativamente do que os homens, embora tenham maior sobrevida. A principal explicação para a pior percepção de saúde das mulheres está relacionada ao seu papel na sociedade, que provoca uma dupla jornada de trabalho, além de sua menor participação no mercado de trabalho remunerado 4,24. Também se verifica, nas mulheres, uma maior prevalência de doenças crônicas e, por vezes, incapacitantes, e que possuem baixa mortalidade, como a artrite e a depressão 25,26 .

Diversos estudos evidenciaram achados de maior sobrevida e menor incidência de problemas de saúde entre indivíduos casados, quando comparados aos não casados $27,28,29$. Isso parece ocorrer mais fortemente entre os homens do que nas mulheres. Diversas hipóteses tentam explicar esse efeito protetor. O principal modelo explicativo é aquele relacionado ao estresse/suporte social 27 . O casamento também pode levar a hábitos de vida mais saudáveis 29. Entretanto, um estudo conduzido na Espanha, numa população de trabalhadores, revelou que ser casado(a), ou morar junto com companheiro(a), não são condições necessariamente relacionadas a melhores níveis de saúde. Os autores sugeriram que, ao se avaliar a associação entre características familiares e saúde, deve-se levar em conta a influência de outras variáveis, como: sexo, nível socioeconômico e contexto sociocultural. Também questionaram que, na maioria dos estudos prévios que revelaram associação positiva entre situação conjugal e saúde, o desenho seccional pode ter favorecido a ocorrência de causalidade reversa nessa associação ${ }^{30}$. Em estudo brasileiro baseado em um inquérito telefônico (Vigilância de Fatores de Risco e Proteção para Doenças Crônicas por Inquérito Telefônico - VIGITEL), também não foram encontradas diferenças significativas nas razões de prevalência de autoavaliação de saúde ruim, segundo estado conjugal (solteiro, casado, viúvo e separado/divorciado) 19. No presente estudo, após o ajuste pelas demais variáveis, as razões de chance foram muito próximas, e não estatisticamente significativas, para os diferentes estratos de situação conjugal.

O estudo VIGITEL também verificou que os indivíduos pertencentes às regiões Norte e Nordeste do Brasil possuem maior prevalência de autoavaliação do estado de saúde ruim. Da mesma forma, no presente estudo, indivíduos não pertencentes a essas regiões, ou seja, aqueles que 
referiram morar nas regiões Centro-oeste, Sul ou Sudeste do país, apresentaram chances significativamente menores de relatar autoavaliação negativa do estado de saúde, do que aqueles residentes na Região Nordeste ${ }^{19}$. Esses achados podem estar relacionados a questões estruturais nessas regiões, ligadas à assistência em saúde mais precária, em comparação às regiões Sul e Sudeste, o que indica a necessidade de maiores investimentos nelas, a fim de melhorar a saúde de suas populações.

Em relação aos achados para a variável raça, após o controle por escolaridade, renda, comportamentos relacionados à saúde, sexo, idade, morbidade e IMC, a associação foi marginalmente significativa e a direção do efeito foi similar à encontrada em outros estudos brasileiros, como o da PNAD e o VIGITEL 18,19. O estudo VIGITEL mostrou uma prevalência de autoavaliação de saúde ruim maior entre homens e mulheres que referiram cor de pele preta, parda ou morena, comparados aos que se autoavaliaram como brancos (razão de prevalência $-\mathrm{RP}=1,15$ em homens e RP = 1,31 em mulheres) 19. Por outro lado, a magnitude da associação entre raça e autoavaliação do estado de saúde foi bem menor que a encontrada nos estudos norte-americanos. Estudo realizado nos Estados Unidos verificou que indivíduos de raça branca tiveram quase $300 \%$ mais chance de autoavaliarem positivamente a sua saúde, quando comparados aos negros 31 . Segundo os autores, esse achado poderia ser explicado por um maior pessimismo que indivíduos da raça negra possuem com relação à sua saúde, ao serem comparados com indivíduos da raça branca e isso seria encontrado nos piores resultados de autoavaliação de saúde. No entanto, Brasil e Estados Unidos apresentam diferenças importantes nas relações étnicas e raciais de suas populações e essa hipótese provavelmente não poderia ser extrapolada para o contexto brasileiro.

A escolaridade apresentou forte associação com autoavaliação do estado de saúde neste estudo, mesmo após o controle pelas demais variáveis. Indivíduos com menor nível de escolaridade apresentaram $300 \%$ mais chance de ter autoavaliação ruim, em relação aos de escolaridade mais alta. A associação entre baixos níveis de renda e autoavaliação de saúde ruim também foi significativa, mesmo após o ajuste. Entre as teorias que buscam explicar os gradientes sociais em saúde destacam-se: a estrutural/materialista, que centraliza as explicações nas condições materiais de vida, a baseada nos comportamentos/ estilo de vida, que enfatiza o papel das opções dos indivíduos, e a psicossocial, que toma por base a teoria do estresse na produção de doen- ça ${ }^{32}$. Mesmo após o ajuste por variáveis relacionadas aos comportamentos em saúde, pela situação conjugal (assumindo-se como proxy do suporte social) e pela renda, um marcador de nível socioeconômico, o efeito da escolaridade ainda foi bastante acentuado, revelando-se um importante mediador da relação entre nível socioeconômico e percepção de saúde, que está de acordo com o observado pela grande importância desse marcador em estudos nacionais e internacionais 4,17,18,19,33. Essa importância se deve a dois aspectos principais. O nível de instrução é a principal causa das desigualdades sociais em saúde porque reflete o capital humano (i.e., as habilidades cognitivas e não cognitivas, que podem controlar ou direcionar a vida de um indivíduo, provendo os recursos necessários, para além dos financeiros) ${ }^{34}$. Em segundo lugar, aqueles com maiores níveis de escolaridade tendem a adotar mais frequentemente hábitos de vida saudáveis, como: a prática de atividade física, a manutenção de peso adequado, o consumo moderado de álcool e o hábito de não fumar 35 . O estudo VIGITEL também verificou que os comportamentos relacionados à saúde podem representar uma mediação importante do efeito da escolaridade na autoavaliação do estado de saúde 19.

A literatura mostra que o tabagismo e o sedentarismo estão associados a uma pior autoavaliação do estado de saúde, conforme estudos já publicados na Finlândia e no Sul do Brasil 13,24. O tabagismo, no presente estudo, mostrou associação com autoavaliação de saúde ruim mesmo após o ajuste pelas outras variáveis. O estudo VIGITEL, conduzido em diferentes capitais brasileiras, encontrou associação entre fumar $20 \mathrm{ou}$ mais cigarros por dia e referir um estado de saúde ruim. Neste estudo, também foi verificada a associação entre autoavaliação de saúde ruim e sedentarismo ${ }^{19}$. No presente estudo, ser sedentário esteve relacionado aproximadamente ao dobro de chance de ter autoavaliação de saúde ruim. Em relação ao consumo de álcool, foram significativos apenas, no modelo ajustado, os resultados referentes à categoria dos indivíduos que relataram não consumir bebida alcoólica, os quais apresentaram maior chance de ter autoavaliação do estado de saúde ruim do que os indivíduos com consumo moderado. Na literatura, existem evidências de que a relação bruta entre o consumo de álcool e a autoavaliação de saúde tenha um padrão de "curva em U", com os indivíduos que bebem moderadamente apresentando melhores percepções de saúde do que os indivíduos nos extremos da categoria. Após o controle por diversas outras variáveis, o padrão dessa associação se aproxima de uma "curva em J" 13. No presente estudo, o padrão em U 
foi claramente encontrado na análise univariada. Após o ajuste, a categoria de indivíduos que relatou não consumir bebida alcoólica teve significativamente maior relato de autoavaliação de saúde ruim, enquanto a categoria de maior consumo de álcool apresentou um resultado apenas marginalmente significativo.

A dimensão física da saúde é considerada, em diversos estudos, o determinante mais importante na autoavaliação do estado de saúde $9,22,23,36,37,38,39$. Neste estudo, também se revelou forte associação entre presença de doença crônica e autoavaliação de saúde, o que está de acordo com resultados nacionais e internacionais 17,19, e a relação observada apresentou um gradiente dose-resposta. Em relação ao IMC, diferentemente de estudo conduzido na Suécia e de um estudo brasileiro, o baixo peso não esteve associado à autoavaliação de saúde ruim na análise ajustada. Já os indivíduos obesos estiveram relacionados à autoavaliação de saúde ruim mesmo após o controle pelas demais variáveis, da mesma forma que nos estudos mencionados 19,32.

A principal limitação deste estudo está relacionada ao seu desenho. Alguns dos fatores analisados, sobretudo os fatores comportamentais ou hábitos de vida, podem ter sofrido o efeito da causalidade reversa e suas análises devem ser feitas com cautela. Outro aspecto importante é a ocorrência do viés de sobrevivência seletiva em estudos seccionais, que decorrem de uma perda (morte) desproporcional dos indivíduos antes da seleção para participação no estudo. Assim, os indivíduos mais saudáveis são os que sobrevivem por mais tempo e, consequentemente, pode haver um resultado subestimado da real magnitude da autoavaliação de saúde ruim na população.

A autoavaliação de saúde, segundo alguns estudos, é considerada um importante fator na predição de problemas de saúde que só seriam detectados mais tardiamente e do uso de serviços de saúde 8,9,40,41. Um estudo que avaliou a importância de diferentes desfechos em saúde no uso de serviços verificou que a autoavaliação de saúde foi o determinante mais importante do uso, independentemente do tipo de serviço de saúde avaliado ${ }^{40}$. Em estudos de utilização dos serviços de saúde, a necessidade percebida costuma estar mais relacionada à procura pelo cuidado em saúde e à adesão ao tratamento, ao passo que a necessidade avaliada relaciona-se mais ao tipo de tratamento que será realizado após a consulta inicial 42 .

A percepção negativa do estado de saúde exerce uma influência na decisão do indivíduo de procurar um médico ou um serviço de saúde. Nesse sentido, os indivíduos com autoavaliação de saúde ruim formarão a demanda dos serviços de saúde ${ }^{41}$. E tais serviços precisam estar preparados para receber e atender a essa demanda. Para isso, é importante conhecer o perfil dos indivíduos mais propensos a buscar os serviços de saúde.

No presente estudo, quanto maior a idade, pior foi a percepção do estado de saúde dos indivíduos. Baixos níveis de escolaridade e renda também se associaram a uma pior autoavaliação de saúde. Esses achados evidenciam a persistência de desigualdades sociais em saúde na população brasileira. A raça e a situação conjugal não estiveram associadas à autoavaliação de saúde ruim. Em contrapartida, a adoção de hábitos de vida não saudáveis ainda é um importante fator associado à autoavaliação de saúde ruim na população, como o tabagismo e o sedentarismo. A presença de doença crônica e de obesidade também estiveram associadas ao desfecho. Acreditase que os resultados obtidos, ao identificarem fatores associados à autoavaliação de saúde ruim, podem contribuir para a identificação das características da população que estão associadas a um pior estado de saúde percebido e, dessa forma, gerar hipóteses a serem testadas por estudos longitudinais. 


\section{Resumen}

La autoevaluación del estado de salud es un importante indicador de la percepción de salud. Este estudio tiene por objetivo investigar la asociación entre autoevaluación de salud y factores sociodemográficos, hábitos de vida y morbilidad en la población brasileña. Se trata de un estudio seccional, basado en una encuesta nacional, que evaluó a 12.324 individuos, en las cinco regiones geográficas del país. Se aplicaron modelos de regresión para evaluar la asociación entre el desenlace, evaluado de forma dicotómica, y variables de exposición: sexo, edad, renta, escolaridad, raza, situación conyugal, tabaquismo, actividad física, consumo de alcohol, presencia de morbilidad crónica e índice de masa corporal. El aumento de la edad, poseer bajos niveles de escolaridad y renta, tabaquismo, sedentarismo, presencia de morbilidad crónica y obesidad fueron los principales factores asociados a una mala autoevaluación. La identificación de las características de la población que están asociadas a un peor estado de salud percibido puede contribuir para trazar un perfil de los individuos más propensos a procurar los servicios de salud.

Autoevaluación Diagnóstica; Morbidad; Factores Epidemiológicos; Encuestas Epidemiológicas

\section{Colaboradores}

A. L. B. Pavão participou da revisão bibliográfica, delineamento do estudo, análise estatística, e redação do artigo. G. L. Werneck contribuiu na supervisão, delineamento do estudo, análise estatística e redação do artigo. M. R. Campos colaborou no delineamento do estudo, análise estatística e redação do artigo.

\section{Agradecimentos}

A. L. B. Pavão recebeu auxílio para a realização da pesquisa mediante bolsa, concedida pelo CNPq, modalidade doutorado no país (no 140191/20097).

\section{Referências}

1. Ferreira $\mathrm{ABH}$. Novo dicionário Aurélio da língua portuguesa. 2a Ed. Rio de Janeiro: Editora Nova Fronteira; 1986.

2. Larson JS. The conceptualization of health. Med Care Res Rev 1999; 56:123-36.

3. Mendoza-Sassi R, Béria JU. Utilización de los servicios de salud: una revisión sistemática sobre los factores relacionados. Cad Saúde Pública 2001; 17:819-32.

4. Szwarcwald CL, Souza-Júnior PRB, Esteves MAP, Damacena GN, Viacava F. Socio-demographic determinants of self-rated health in Brazil. Cad Saúde Pública 2005; 21 Suppl 1:S54-64.

5. Pikhart H, Bobak M, Siegrist J, Pajak A, Rywik S, Kyshegyi J, et al. Psychosocial work characteristics and self rated health in four post-communist countries. J Epidemiol Community Health 2001; 55:624-30.

6. Franks P, Gold MR, Fiscella K. Sociodemographics, self-rated health, and mortality in the US. Soc Sci Med 2003; 56:2505-14.
7. Hunt SM, McKenna SP, McEwen J, Backett EM, Williams J, Papp E. A quantitative approach to perceived health status: a validation study. J Epidemiol Community Health 1980; 34:281-6.

8. Blank N, Diderichsen F. The prediction of different experiences of long-term illness: a longitudinal approach in Sweden. J Epidemiol Community Health 1996; 50:156-61.

9. Kaplan GA, Goldberg DE, Everson SA, Cohen RD, Salonen R, Tuomilehto J, et al. Perceived health status and morbidity and mortality: evidence from the Kuopio ischaemic heart disease risk factor study. Int J Epidemiol 1996; 25:259-65.

10. Borg V, Kristensen TS. Social class and self-rated health: can the gradient be explained by differences in life style or work environment? Soc Sci Med 2000; 51:1019-30.

11. Ferrie JE, Shipley MJ, Marmot MG, Stansfeld S, Smith GD. Health effects of anticipation of job change and non-employment: longitudinal data from the Whitehall II study. BMJ 1995; 311:1264-9. 
12. Wolinsky FD, Stump TE, Clark DO. Antecedents and consequences of physical activity and exercise among older adults. Gerontologist 1995; 35:451-62.

13. Poikolainen K, Vartianen E, Korhonen HJ. Alcohol intake and subjective health. Am J Epidemiol 1996; 144:346-50.

14. House JS, Landis KR, Umberson D. Social relationships and health. Science 1988; 241:540-5.

15. Berkman LF, Syme L. Social networks, host resistance, and mortality: a nine year follow-up study of Alameda county residents. Am J Epidemiol 1979; 109:176-204.

16. Kaplan BH, Cassel JC, Gore S. Social support and health. Med Care 1977; 15:47-58.

17. Cott CA, Gignac MAM, Badley EM. Determinants of self rated health for Canadians with chronic disease and disability. J Epidemiol Community Health 1999; 53:731-6.

18. Dachs JNW, Santos APR. Autoavaliação do estado de saúde no Brasil: análise dos dados da PNAD/2003. Ciênc Saúde Coletiva 2006; 11:887-94.

19. Barros ABA, Zanchetta LM, Moura EC, Malta DC. Autoavaliação de saúde e fatores associados, Brasil, 2006. Rev Saúde Pública 2009; 43 Suppl 2:27-37.

20. Szwarcwald CL, Damacena GN. Amostras complexas em inquéritos populacionais: planejamento e implicações na análise estatística dos dados. Rev Bras Epidemiol 2008; 11 Suppl 1:38-45.

21. Alazraqui M, Roux AVD, Fleischer N, Spinelli H. Salud auto-referida y desigualdades sociales, ciudad de Buenos Aires, Argentina, 2005. Cad Saúde Pública 2009; 25:1990-2000.

22. Damian J, Ruigomez A, Pastor V, Martin-Moreno JM. Determinants of self-assessed health among Spanish older people living at home. J Epidemiol Community Health 1999; 53:412-6.

23. Shooshtari S, Menec V, Tate R. Comparing predictors of positive and negative self-rated health between younger (25-54) and older (55+) Canadian adults: a longitudinal study of well-being. Res Aging 2007; 29:512-55.

24. Hofelmann DA, Blank N. Autoavaliação de saúde entre trabalhadores de uma indústria no sul do Brasil. Rev Saúde Pública 2007; 41:777-87.

25. Denton M, Walters V. Gender differences in structural and behavioral determinants of health: an analysis of the social production of health. Soc Sci Med 1999; 48:1221-35.

26. Ross CE, Bird CE. Sex stratification and health lifestyle: consequences for men's and women's perceived health. J Health Soc Behav 1994; 35:161-78.

27. Burman B, Margolin G. Analysis of the association between marital relationships and health problems: an interactional perspective. Psychol Bull 1992; 112:39-63.

28. Johnson NJ, Backlund E, Sorlie PD, Loveless CA Marital status and mortality: the National Longitudinal Mortality Study. Ann Epidemiol 2000; 10:224-38.
29. Lillard LA, Panis CWA. Marital status and mortality: the role of health. Demography 1996; 33:313-27.

30. Artazcoz L, Cortès I, Borrell C, Escribà-Agüir V, Cascant L. Social inequalities in the association between partner/marital status and health among workers in Spain. Soc Sci Med 2011; 72:600-7.

31. Spencer SM, Schulz R, Rooks RN, Albert SM, Thorpe Jr. RJ, Brenes GA, et al. Racial differences in self-rated health at similar levels of physical functioning: an examination of health pessimism in the Health, Aging, and Body Composition Study. J Gerontol B Psychol Sci Soc Sci 2009; 64:87-94.

32. Molarius A, Berglund K, Eriksson C, Lambe M, Nordstrom E, Eriksson HG, et al. Socioeconomic conditions, lifestyle factors, and self-rated health among men and women in Sweden. Eur J Public Health 2006; 17:125-33.

33. Cavelaars AE, Kunst AE, Geurts JJ, Crialesi R, Grotvedt L, Helmert U, et al. Differences in self-reported morbidity by educational level: a comparison of 11 western European countries. J Epidemiol Community Health 1998; 52:219-27.

34. Zhang W, McCubbin H, McCubbin L, Chen Q, Foley S, Strom I, et al. Education and self-rated health: an individual and neighborhood level analysis of Asian Americans, Hawaiians, and Caucasians in Hawaii. Soc Sci Med 2010; 70:561-9.

35. Ross CE, Wu C. The links between education and health. Am Sociol Rev 1995; 60:719-45.

36. Benyamini Y, Leventhal EA, Leventhal H. Gender differences in processing information in making self-assessments of health. Psychosom Med 2000; 62:354-64.

37. Blaum CS, Liang J, Liu X. The relationship of chronic diseases and health status to the health services utilization of older Americans. J Am Geriatr Soc 1994; 42:1087-93

38. Ferraro KF. Self-ratings of health among the old and the old-old. J Health Soc Behav 1980; 21:377-83.

39. Idler EL, Hudson SV, Leventhal H. The meanings of self-ratings of health. A quantitative and qualitative approach. Res Aging 1999; 21:458-76.

40. Fylkesnes K. Determinants of health care utilization: visits and referrals. Scand J Soc Med 1993; 21:40-50.

41. Hulka BS, Wheat JR. Patterns of utilization: the patient perspective. Med Care 1985; 23:438-60.

42. Andersen RM. Revisiting the behavioral model and access to medical care: does it matter? J Health Soc Behav 1995; 36:1-10.

Recebido em 19/Jun/2012

Versão final reapresentada em 23/Nov/2012

Aprovado em 06/Dez/2012 